\title{
Multi-agent-based Cooperative Control for Hybrid Renewable Energy Generation System
}

\author{
Wang Qiang ${ }^{1,}$ a and Chen Jun ${ }^{2, b}$ \\ ${ }^{1}$ College of Electrical Engineering \& New Energy, China Three Gorges University, Yichang, China \\ ${ }^{2}$ Power Supply of Chongren County, Jiangxi Province, China \\ awq00518@sohu.com, ${ }^{\mathrm{b}}$ chenjun@126.com
}

\begin{abstract}
Keywords: Hybrid renewable energy generation system; Multi-agent; Cooperative control; Particle swarm algorithm.

Abstract. A multi-agent-based cooperative control strategy (MACCS) is proposed for hybrid renewable energy generation system with large-scale wind-photovoltaic energy storage units (WPSUs). The proposed system adopts the negotiation model of the contract net protocol with the non-fixed client-server cooperative mechanism among agents. In the cooperative control process, the proposed system solves the global optimal-energy distribution plan of the system by considering the self-constraint and control objective of each agent and by using the improved particle swarm algorithm to achieve the maximum economic benefit on the basis of stable operations. A case is given to discuss the behavior characteristics of the agent and communication negotiation process and to verify that the system designed in this paper can increase the overall economy of the system.
\end{abstract}

\section{Introduction}

The development of renewable energies such as wind power and photovoltaic power has attracted many experts to study its cooperative control systems. However, the operation process of large-scale hybrid renewable power-generation system often suffer from poor stability, and lack of energy-coordination control strategies. Various countries around the world have successively conducted a number of demonstration projects showing improved intermittent power controllability by using energy storage technology[1,2,3,4].

With the increasing construction of wind farms and photovoltaic arrays, as well as the increasing adoption of composite energy storage systems, the defects of the centralized energy control method in flexibility and extensibility hinders the operations of large-scale integrated power-generation systems for wind, photovoltaic, and energy storage units. The multi-agent system, which responds intelligently and flexibly to changes in working conditions and requirements, has been widely applied in various aspects of the power system[5,6,7].

The proposed multi-agent-based cooperative control system simulates the bidding mechanism in the market by using the contract network protocol. By considering itself constraint and interests, the proposed system solves the global optimal-energy distribution plan of the system by the improved particle swarm algorithm to achieve the maximum economic benefit on the basis of stable operations.

\section{The structure of the MACCS}

For the WPSUs, four Agents are designed in the paper, including Wind Turbine (WT) Agent, Photovoltaic (PV) Agent, Battery (BAT) Agent and Load (LD) Agent. Each Agent can control several generating unit of the same characteristics and determine its own control objective according to the characteristics of the equipment. The structure of MA-ECCS is shown in Fig. 1.

The system adopts the non-fixed client-server cooperative mechanism. Facing the conditions such as changes of the demand of the power grid and the meteorological, the joining or quitting of the units, and the imbalanced supply and demand between the power generation unit and the power grid, the corresponding Agent should initiate the energy coordination task as the control center to ensure the stable grid-connected operation of the system. The Agent initiating the energy coordination task is 
considered to be the Task Agent. Other Agents determine how to participate in the energy coordination according to their own characters and control objectives. In order to avoid the conflict caused by several Agents initiating the energy coordination tasks at the same moment, the timestamp Task- Token is set to be the symbol of Task Agent. Only the Task Agent with Task-Token in the effective period of time can initiate the energy coordination task.

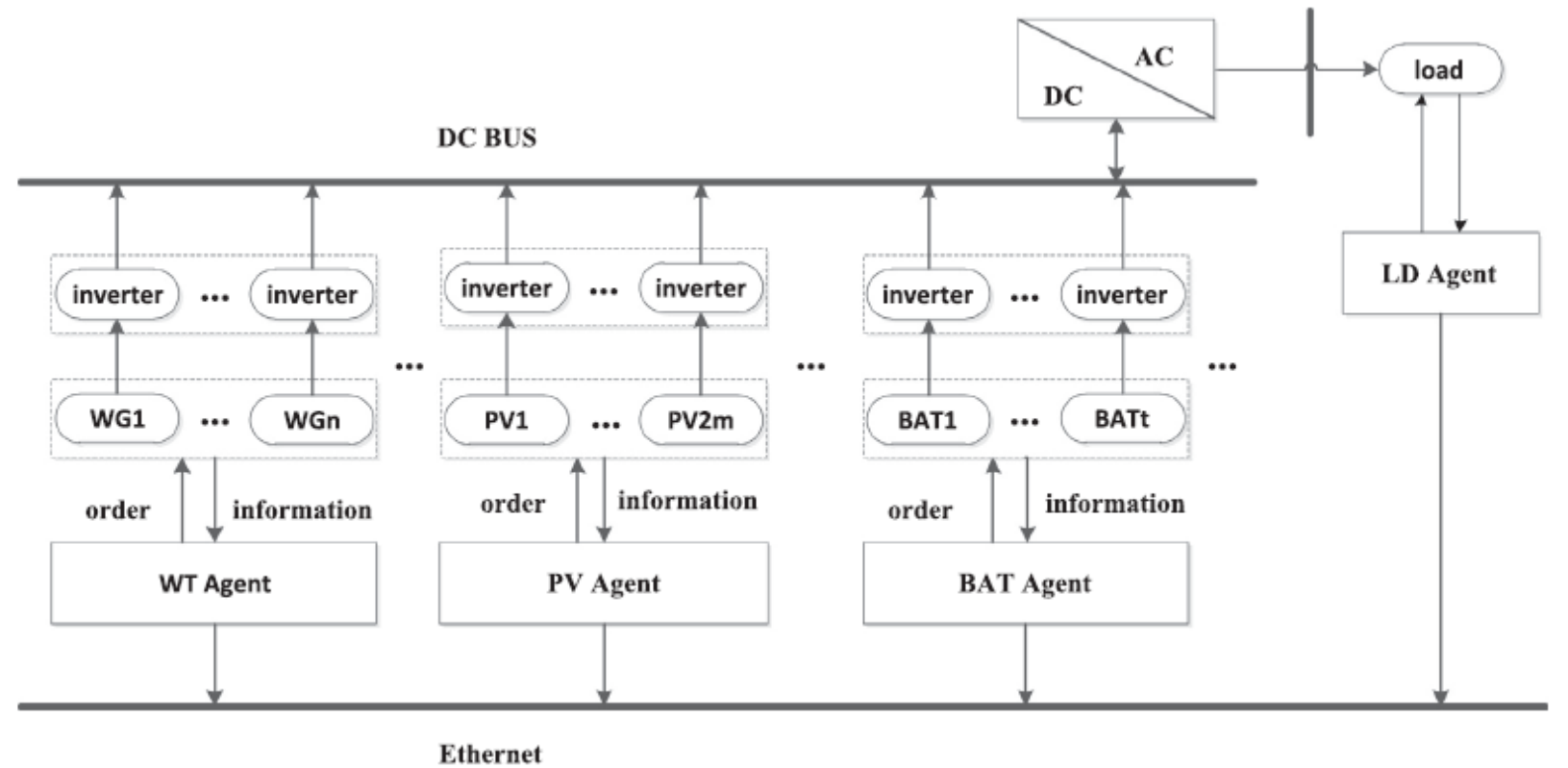

Fig.1 The structure of MACCS

\section{Agent characteristic analysis}

WT Agent and PV Agent. WT Agent and PV Agent represent the control units of the wind turbine and the photovoltaic panel, respectively. As the wind turbine and the photovoltaic panel operate at a low levelized cost, in the principle of maximizing the use of renewable energy, the characteristics of WT Agent and PV Agent are designed to be participating in the bidding actively until all the available equipment operate to the maximum power output. At the same time, they can initiate the energy coordination task when the power output changes.

The output power characteristics of the wind generator can be expressed as [8]:

$$
P_{W T}(k)=\left\{\begin{array}{cc}
0 & v(k)<v_{\min } \text { or } v(k)>v_{\max } \\
P_{\text {rated }} & v_{\text {rated }} \leq v(k) \leq v_{\max } \\
P_{\text {rated }} \frac{v(k)^{2}-v_{\min }^{2}}{v_{\text {rated }}^{2}-v_{\min }^{2}} & v_{\min } \leq v(k)<v_{\text {rated }}
\end{array}\right.
$$

Where $P_{W T}(k)$ is the output power of the wind generator at time $k ; P_{\text {rated }}$ is the rated power of the wind generator; $v_{\min }, v_{\max }$, and $v_{\text {rated }}$ are the minimum start-up wind speed, cutoff wind speed, and minimum rated wind speed of the wind generator.

Battery Agent. BAT Agent in the system can monitor the state of charge (SOC) of the battery and manage the charge and discharge of the batteries. It can be divided into the following two types according to its response characteristics: the control objective of the first one, ECO-BAT Agent, is to maximize economic benefits. When responding to the energy coordination task initiated by Task Agent, ECO-BAT Agent will take full consideration to the price information of the current load demand and determine the bid value B according to its own SOC state. It does not receive the change of Task Agent; the control objective of the second one, EME-BAT Agent, is to maximize the power supply reliability. When responding to the energy coordination task, it will consider the current SOC 
state and then determine the maximum charge and discharge interval as the bid value $\mathrm{B}$. At the same time, it receives any valid value in the interval coordinated by Task Agent.

The output power model of the battery is [8]:

$$
\begin{aligned}
& \text { Charge: } \quad \operatorname{SOC}(t+1)=\operatorname{SOC}(t) \cdot(1-\sigma)+\frac{P_{C H}(t) \cdot \eta_{C H}}{E_{\max }} \\
& \text { Discharge: } \quad \operatorname{SOC}(t+1)=\operatorname{SOC}(t) \cdot(1-\sigma)-\frac{P_{D I S}(t) \cdot \Delta t}{E_{\max } \eta_{D I S}} \Delta t
\end{aligned}
$$

$S O C(t)$ is the battery state of charge after $t ; \sigma$ is the self discharge per hour of the battery; $P_{C H}$ and $P_{D I S}$ are the charge and discharge powers of the battery in $t$, respectively; $\Delta t$ is the length of $t ; \eta_{C H}$ and $\eta_{D I S}$ are the charge and discharge efficiencies of the battery, respectively; and $E_{\max }$ is the maximum capacity of the battery.

LD Agent. With satisfying the load demand of the power grid as the control objective, LD Agent can make the real-time monitoring of the change of the load and classify the load priorities according to the importance and can cut the less important load when the power generation unit cannot meet the load demand of the power grid. After the global energy coordination by Task Agent, if the returned bid value $\mathrm{R}$ is 0 , it suggests the balanced energy coordination; if the returned bid value $\mathrm{R}$ is greater than zero, it shows that the power generation unit cannot meet the load demand of the power grid.

\section{Objective and strategies of global coordination control}

Objective function. The objective of the WPS-PGUs is to maximize the overall economic benefit of the system $\mathrm{F}$ in the study period $\mathrm{T}$ :

$$
\max F=P_{\text {sub }} \int_{0}^{T} P d t-\left[C_{O P}(P)+C_{D E P}(P)+C_{P U N}\right]
$$

Where $P$ refers to the total output of the power generation unit; $P_{s u b}$ is the Feed-In Tariff; $C_{O P}(P)$, $C_{D E P}(P)$ and $C_{P U N}$ refer to the overall operation cost and depreciation cost of the equipment and the penalty cost for load cutting in the research period, respectively.

The global cooperative control algorithm. The particle swarm algorithm is used in the paper to solve the global optimal energy allocation plan. However, traditional particle swarm optimization may fall into local optimal solution due to premature convergence during the iteration process. The paper improves the search efficiency and the global search ability of the algorithm through improving the aspects such as inertia weight, learning factor and mutation particle.

\section{Case study}

Simulation environment. The structure of the WPS-PGUs case study designed in the paper is shown in Fig. 2, in which WG1 to WG4 represent the single wind turbines; PU1 to PU4 represent one photovoltaic unit is formed by connecting several photovoltaic panels of the same type to the independent inverter chamber through the feeder. BAT1 to BAT4 represent one battery energy storage unit is formed by connecting several battery cabinets into a battery pack series and then connecting other battery pack series in parallel. BAT1 and BAT2 are the vanadium flow batteries which are controlled by ECO-BAT; BAT3 and BAT4 are the phosphoric acid iron batteries which are controlled by EME-BAT. The total installed capacities of wind/photovoltaic/energy storage are $9 \mathrm{MW} / 8.16$ MW/5 MW, respectively.

Results and analysis. According to the calculation results in Table 1, the MAS cooperative control system can reduce the operation cost and depreciation cost of the system. This part of costs is used to prolong the service life of the batteries on the basis of optimizing the charging and discharging strategies of the batteries to increase the overall economy of the system. By contrast, the profit of the method proposed in the paper increases by $16.4 \%$. 


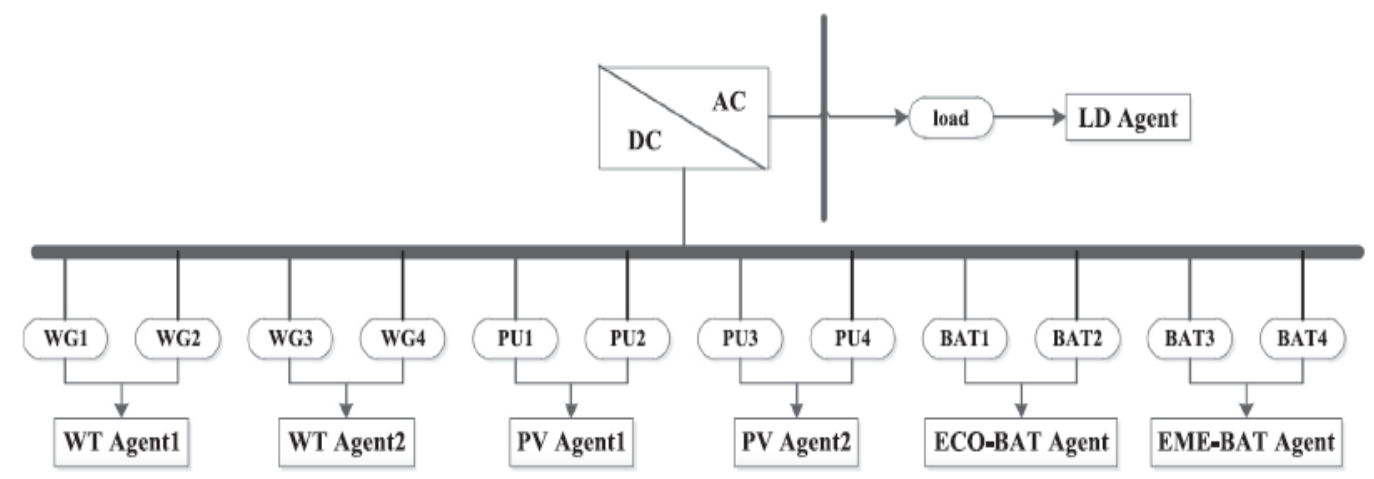

Fig. 2. The structure of the WPS-PGUs case study

Table 1 Economic indicators of two models

\begin{tabular}{llllll}
\hline Scheme & Income(US\$) & $\begin{array}{l}\text { Operating } \\
\text { cost(US\$) }\end{array}$ & $\begin{array}{l}\text { Depreciation } \\
\text { expense(US\$) }\end{array}$ & $\begin{array}{l}\text { Punishment } \\
\operatorname{cost}(\text { US\$) }\end{array}$ & Earning(US\$) \\
\hline Scheme 1 & 37,560 & 237.5 & 14,996 & 0 & $22,356.5$ \\
Scheme 2 & 37,560 & 166.7 & 11,372 & 0 & $26,021.3$ \\
\hline
\end{tabular}

\section{Conclusions}

A multi-agent-based cooperative control system for large-scale wind, photovoltaic, energy storage, and power-generation units is designed in this study. By building on the non-fixed client-server cooperative mechanism in the distributed environment, the system enhances flexibility and extensibility, avoids the single point of failure in the centralized control system, and increases operation stability. The proposed system uses the contract network protocol as the communication mechanism among agents and solves the global optimal-energy distribution plan by using the improved particle swarm algorithm by considering the self-constraints and interests of each part.

\section{References}

[1] Campoccia, A., and E., Zizzo, G., Comparative analysis of different supporting measures for the production of electrical energy by solar PV and wind systems: four representative European cases. Sol. Energy 83 (3), p.287

[2] Askarzadeh, A., Developing a discrete harmony search algorithm for size optimization of wind-photovoltaic hybrid energy system. Sol. Energy 98, p.190

[3] Merei, G., Berger, C., Sauer, D.U., Optimization of an off-grid hybrid PV-wind-diesel system with different battery technologies using genetic algorithm. Sol. Energy 97, p.460

[4] Bayod-Ru' jula, Sizing criteria of hybrid photovoltaic-wind systems with battery storage and self-consumption considering interaction with the grid.Sol. Energy 98, p.582

[5] Kremers, E., Gonzalez de Durana, J., Barambones, O., Multi-agent modeling for the simulation of a simple smart microgrid. Energy Convers. Manage. 75, p.643

[6] da Rosa, M.A., Leite da Silva, A.M., Miranda, V., Multi-agent systems applied to reliability assessment of power systems. Int. J. Electr. Power Energy Syst. 42 (1), p.367

[7] Pipattanasomporn, M., Feroze, H., Rahman, S., Securing critical loads in a PV-based microgrid with a multi-agent system. Renew. Energy 39 (1), p.166

[8] Yang, H., Wei, Z., Chengzhi, L.,Optimal design and technoeconomic analysis of a hybrid solar-wind power generation system. Appl. Energy 86 (2), p.163 